marginal zone and follicular B cells induces B-cell secretion of interleukin (IL)-10, and subsequent secretion of IL-10 by $\mathrm{T}$ cells. In a mouse model of collagen-induced arthritis, injection of ACs before the onset of clinical disease resulted in lower disease activity than that seen in control mice; this was also true for mice injected with $B$ cells, but not $T$ cells, isolated from AC-treated mice. Further investigation revealed increased IL-10 secretion by antigenspecific $\mathrm{T}$ cells and reduced levels of pathogenic autoantibodies in the AC-treated arthritic mice. The protective effect of AC treatment in vivo was negated by blockade of IL-10 action, indicating that IL-10 is essential in AC-induced $B-c e l l$ protection from autoimmune-mediated inflammation and bone destruction.

The authors conclude that direct interaction between ACs and B cells provides protection from autoimmune disease. Inappropriate $\mathrm{B}$-cell responses to ACs might, therefore, mediate tolerance failure associated with these diseases.

Original article Gray M et al. (2007) Apoptotic cells protect mice from autoimmune inflammation by the induction of regulatory B cells. Proc Natl Acad Sci USA 104: 14080-14085

\section{Twin study shows that lifting force and body weight affect BMD}

The estimated influence of genetic heritability on bone mineral density (BMD) varies markedly; intergenerational studies have attributed 50$70 \%$ of BMD variation to genetic factors, and twin studies have suggested a total heredity of 80-90\%. These estimates are affected by adjusting for the heritability of other factors related to $\mathrm{BMD}$, such as lean body mass and leg strength; therefore, Videman and colleagues conducted a twin study to evaluate the influences of heritability and anthropometric factors on BMD of the femoral neck and lumbar spine. The study included data from a representative sample of 147 monozygotic and 153 dizygotic twins (age 35-70 years) from the Finnish Twin Cohort.

Genetic heritability was estimated to account for $75 \%$ of the variance of femoral neck BMD and $83 \%$ of lumbar spine BMD $\left(L_{1}-L_{4}\right.$ lumbar vertebrae). Isokinetic lifting force and lean body mass/height significantly influenced the heritability of BMD. Lifting force explained $9 \%$ and lean body mass/height explained $18 \%$ of the total genetic variation of femoral BMD; for lumbar spine BMD, these proportions were $9 \%$ and $11 \%$ for lifting force and lean body weight/height, respectively.

These results indicate that body weight was important only for lumbar BMD, while lifting force accounted for an important portion of both femoral and lumbar BMD. The authors conclude that muscle-strengthening exercises might have more practical importance for BMD modification (particularly at the femoral neck) than other life-style factors.

Original article Videman T et al. (2007) Heritability of BMD of femoral neck and lumbar spine: a multivariate twin study of Finnish men. J Bone Miner Res 22: 1455-1462

\section{Symptoms of depression are common among patients with systemic sclerosis}

Rates of depression among patients with chronic diseases are typically several times higher than those reported for the general population. Patients with systemic sclerosis (SSc) are at particular risk of depression because the disease is characterized by chronic pain, fatigue and, frequently, disfigurement. Thombs et al. carried out a systematic review to assess the prevalence of depression, the course of depression and the factors that predict depression in patients with SSc.

Searches of the MEDLINE, CINAHL and PsycINFO databases and hand searches of 26 selected journals were conducted for original articles in any language that used a standardized interview or validated questionnaire to assess depression in patients with SSc. Eight full-text articles published between 1996 and 2006 that included 551 patients in total were eligible for inclusion in the systematic review; all studies assessed prevalence of symptoms of depression but none used a clinical interview for this purpose.

Symptoms of depression were common among patients with SSc; in the eight studies analyzed, $36-65 \%$ of patients were found to have clinically significant symptoms of depression. These depression rates are high compared with those reported for other groups of patients with chronic conditions when the same assessment tools and score cutoffs were used. Studies that reported the course 